\title{
Selection of adequate optimization criteria in chromatographic separations
}

\author{
P. F. Vanbel • P. J. Schoenmakers
}

Received: 2 November 2008 / Revised: 9 February 2009 / Accepted: 12 February 2009 /Published online: 19 March 2009

(C) The Author(s) 2009. This article is published with open access at Springerlink.com

\begin{abstract}
Computer-assisted optimization of chromatographic separations is still a fruitful activity. In fact, advances in computerized data handling should make the application of systematic optimization strategies much easier. However, in most contemporary applications, the optimization criterion is not considered to be a key issue (Vanbel, J Pharm Biomed, 21:603-610, 1999). In this paper, an update of the importance of selecting adequate criteria in chromatographic separation is presented.
\end{abstract}

Keywords Optimization criteria $\cdot$ HPLC separation . Two-dimensional chromatography $\cdot$ Multicriteria decision techniques $\cdot$ Chemometrics

\section{Introduction}

Many different criteria have been suggested in the literature to assess the quality of chromatographic separations. [1-5] Optimization criteria that adequately describe the quality of the separation are among the essential factors determining the applicability of selectivity-optimization procedures. Indeed, the result of an optimization process depends on the selected optimization criteria, so that the latter have to be defined in the context of the objectives of the separation.

P. F. Vanbel • P. J. Schoenmakers

Polymer-Analysis Group,

Van't Hoff Institute for Molecular Sciences (HIMS),

Faculty of Science, University of Amsterdam,

Nieuwe Achtergracht 166,

1018 WV Amsterdam, The Netherlands

P. F. Vanbel $(\bowtie)$

Humont 21,

B-7901 Thieulain, Belgium

e-mail: pascale.vanbel@bluewin.ch
In drug analysis, examples of optimization criteria adapted to the separation of selected target analyte(s) from irrelevant solute(s) will be discussed. An important application of such an approach concerns the separation of an active ingredient and its impurities or degradation products from matrix constituents [6].

Considering robustness as an objective from the beginning of method development reduces significantly the chance of failure during the validation process. Robustness of the separation can be included in optimization strategies by using robustness criteria. As robustness cannot be a goal in itself in method development procedure, it must be combined with other quality criteria (e.g., resolution and/or analysis time). Multicriteria decision making (MCDM) techniques are required. Practical examples will be given in this paper $[7,8]$.

Criteria adapted to two-dimensional chromatography (gas chromatography (GC-GC) and liquid chromatography (LC-LC)) [5] will also be discussed. MCDM approaches are a new concept for these techniques.

Currently, the use of complex evolutionary algorithm and computer simulation $[4,9,10]$ are the rule. A review [11] gives details on the mathematical treatment for each particular optimization strategy (total, partial, and specific strategies, deconvolution-oriented optimization and robustness). We will demonstrate that it is not always necessary.

\section{Experimental}

Chromatographic conditions and optimization procedures

In this paper, the two sets of experimental data were already reported in previous articles [6, 7]. The first set of data [7] concerned a mixture of four acidic solutes (benzoic acid, $m$ - 
nitrobenzoic acid, 3,5-dinitrobenzoic acid, and salicylic acid). However, one additional solute (aspirin) is included here. A reversed-phase liquid chromatography was chosen for this study. A 5- $\mu \mathrm{m}_{18}$ LiChrospher column (125× $4 \mathrm{~mm}$ I.D.) and a $5-\mu \mathrm{m} \mathrm{C}_{18}$ LiChrospher precolumn $(4 \times$ $4 \mathrm{~mm}$ I.D.) from Merck (Darmstadt, Germany) were used. The flow rate was $1.0 \mathrm{ml} / \mathrm{min}$, and UV detection was performed at $254 \mathrm{~nm}$.

A $4 \times 3$ experimental design (three levels of methanol volume fraction and four levels of $\mathrm{pH}$ ) was used to realize the simultaneous optimization of $\mathrm{pH}$ and solvent composition (Fig. 1). The volume fraction of $\mathrm{MeOH}(\varphi)$ was varied between 0.30 and $0.40 . \mathrm{pH}$ was varied between 2.76 and 6.83 with a constant total ionic strength of $0.05 \mathrm{M}$.

A sigmoidal model (Eq. 1) was used for describing capacity factors $(k)$ :

$k=\frac{k_{H A}^{\circ} \times e^{S_{H A} \varphi+T_{H A} \varphi^{2}} \times 10^{-\mathrm{pH}}+k_{A^{-}}^{\circ} \times K_{a}^{\circ} \times e^{\left(S_{A^{-}}+Q_{1}\right) \varphi+\left(T_{A^{-}}+Q_{2}\right) \varphi^{2}}}{10^{-\mathrm{pH}}+K_{a}^{\circ} \times e^{\left(Q_{1} \varphi+Q_{2} \varphi^{2}\right)}}$

where $k_{H A}^{\circ}$ and $k_{A^{-}}^{\circ}$ are extrapolated capacity factors of, respectively, the acid and the basic forms of the solute in pure water, $K_{a}^{\circ}$ is the extrapolated acid-dissociation constant in pure water, $S_{H A}$ and $T_{H A}$ are parameters describing the variation of retention with $\varphi$ for acid species, $S_{A^{-}}$and $T_{A^{-}}$ are corresponding parameters for basic species, and $Q_{1}$ and $Q_{2}$ are coefficients describing the variation of the aciddissociation constant with $\varphi$.

Equation 1 was also used to model peak heights and peak areas.

A quadratic expression (Eq. 2) was used to model the asymmetry factors $\left(A_{S}\right)$ :

$$
\begin{aligned}
A_{S}= & b_{0} \times e^{s_{0} \varphi+T_{0} \varphi^{2}}+b_{1} \times e^{\left(s_{1} \varphi+T_{1} \varphi^{2}\right)} \times \mathrm{pH}+b_{2} \\
& \times e^{\left(s_{2} \varphi+T_{2} \varphi^{2}\right)} \times \mathrm{pH}^{2}
\end{aligned}
$$

A detailed description of the chromatographic conditions and optimization procedure can be found in [7].

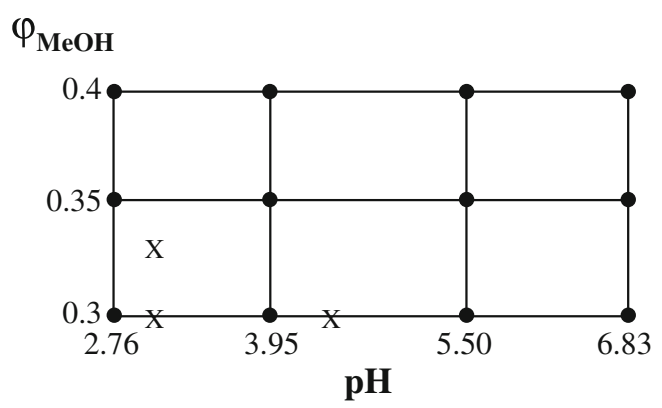

Fig. 1 The $4 \times 3$ experimental design used for the separation of a mixture of five acids. $X$ refers to additional experiments
The second set of data concerned a mixture of nine benzodiazepines (demoxepam, nitrazepam, oxazepam, clonazepam, flunitrazepam, triazolam, nordazepam, diazepam, and ethyl loflazepate). A reversed-phase system was chosen for this study. A 5- $\mu \mathrm{m} 60$ RP-Select B LiChrospher column (125×4 mm I.D.) and a 5- $\mu \mathrm{m} 60$ RP-Select B LiChrospher precolumn $(4 \times 4 \mathrm{~mm}$ I.D.) from Merck (Darmstadt, Germany) were used. The temperature of the column was maintained at $35{ }^{\circ} \mathrm{C}$. The flow-rate was $1.0 \mathrm{ml} / \mathrm{min}$. Chromatograms were recorded at $230 \mathrm{~nm}$. A conventional procedure was used for optimizing the volume fraction of several organic modifiers in the mobile phase, i.e., acetonitrile $(\mathrm{ACN})$, methanol $(\mathrm{MeOH})$ and tetrahydrofuran (THF). The experimental design is shown in Fig. 2. An exponentially quadratic equation (Eq. 3) was used to model the capacity factor $(k)$ as a function of the mobile phase composition:

$k=a_{1} \times e^{a_{2} \times \bar{\varphi}_{1}+a_{3} \times \bar{\varphi}_{2}+a_{4} \times \bar{\varphi}_{1}^{2}+a_{5} \times \bar{\varphi}_{2}^{2}+a_{6} \times \bar{\varphi}_{1} \times \bar{\varphi}_{2}}$

where $\bar{\varphi}_{1}$ is the volume fraction of one solvent (i.e., the binary mixture $\mathrm{ACN} / \mathrm{H}_{2} \mathrm{O}$ ) in the mobile phase and $\bar{\varphi}_{2}$ is the volume fraction of a second solvent (i.e., the binary mixture $\mathrm{MeOH} / \mathrm{H}_{2} \mathrm{O}$ ).

Software

Different "in-house" software programs were developed to model capacity factors and other chromatographic parameters (peak heights, peak areas, and asymmetry factors) and to generate response surfaces. Software is also available for generating predicted or simulated chromatograms. All the programs were written in Pascal (Turbo Pascal 7.0, Borland International, Scotts Valley, CA, USA) and implemented on an IBM-compatible computer. Data generated by Pascal programs were imported directly in

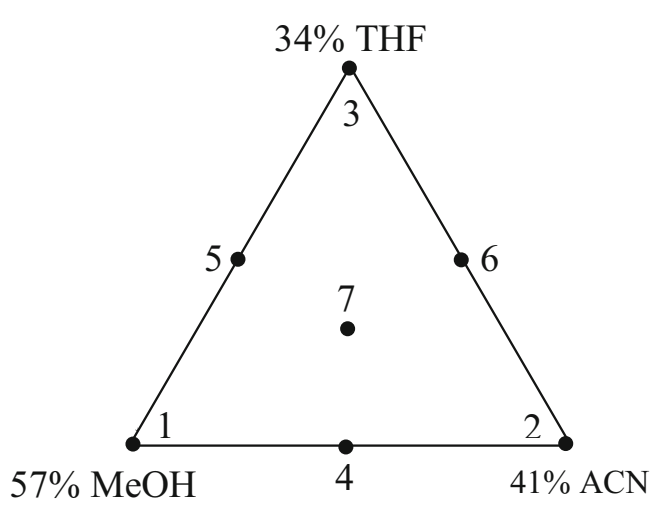

Fig. 2 Experimental design used for the separation of a limited number of solutes from a mixture of nine benzodiazepines 
Excel software (version 5.0) in a Windows environment (Microsoft Corporation) for further manipulation and graphical presentation.

\section{Results and discussion}

High-performance liquid chromatography

There are fundamentally two types of criteria, namely elementary criteria describing the separation between two adjacent peaks and overall criteria that can be used to describe the quality of an entire chromatogram (Tables 1 and 2). These criteria are used for the separation of all peaks in the chromatogram. Criteria for limited optimization have been described in reference [6]. Limited optimization refers to situations in which the chromatographer is interested to separate only some components (relevant solutes) from a matrix of other (irrelevant peaks). Figure 3 illustrates two possible situations. In this figure, $R$ refers to a relevant peak and $I$ to an irrelevant one. In Fig. 3a, we have one relevant peak surrounded by two irrelevant ones. Peaks 1 and 3 (both irrelevant) have no relevant value. The relevant peak (peak 2) has two relevant values of the effective resolution: ${ }^{2} R_{p}$ referring to the separation between peak 2 and the previous one and ${ }^{2} R_{n}$ referring to the separation from the next peak. In Fig. 3 b, peak 1 is relevant and has one relevant value, ${ }^{1} R_{n}$, referring to the separation between peak 1 and the next one. Peak 2 has no relevant value. For peak $3,{ }^{3} R_{p}$ and ${ }^{3} R_{n}$ are both relevant. For peak $4,{ }^{4} R_{p}$ referring to the separation between this peak and the previous one is relevant. As ${ }^{3} R_{n}$ and ${ }^{4} R_{p}$ refer both to the separation between peaks 3 and 4, only the lowest of these two values will be kept (as in complete optimization). $R_{1, \text { min }}$ is the lowest relevant value of $R_{1}$. This type of chromatograms is often obtained in high-performance liquid chromatography (HPLC) and in ion exchange chromatography.

Table 1 Elementary criteria factor, $N$ number of theoretical
$t_{R}$ retention time, $k$ capacity plates, $A_{S}$ asymmetry factor, $h$ peak height

\section{The 1 Elementary citeria}

\footnotetext{
heak height
}

For the threshold resolution (second equation in Table 2) and for the minimum required analysis times and timecorrected resolution products (fifth to eighth equations in Table 2), the analysis time is always determined by the capacity factor of the last peak, whether or not this one is relevant. The minimum resolution value is the lowest relevant value of $S, R_{S}$, or $R_{1}$.

Figure 4 illustrates the importance of considering the relevance of the peaks in practice. The minimum effective resolution is the selected criterion. When all five solutes are relevant, the chromatogram presented in Fig. 4a is selected as optimum by the minimum effective resolution $\left(R_{1, \min }=\right.$ 1.2). However, if the chromatographer is only interested in the separation of two solutes among the five (i.e., benzoic acid and dinitrobenzoic acid), another chromatogram is the optimum (Fig. 4b). The minimum effective resolution is selected from the relevant pairs of peaks. Irrelevant peaks do not need to be separated from each other. Compared to the complete optimization process (Fig. 4a), the value of $R_{1, \text { min }}$ is higher (2.95), and the analysis time is about $6 \mathrm{~min}$ instead of $12 \mathrm{~min}$. Fig. 4c is the optimum chromatogram when dinitrobenzoic is the only interesting peak. This example is particularly favorable. $R_{1, \min }$ is equal to 5.1 , and the analysis time is lower than $5 \mathrm{~min}$. This is due to the fact that irrelevant peaks appear at the beginning of the chromatogram and are almost all confounded. These examples illustrate clearly the interest of considering the relevance of the peaks during an optimization process.

Other specific objectives can be achieved by using particular optimization criteria. So, the calibrated normalized resolution product, $\underline{r}^{*}$ (Eq. 4) promotes situations where relevant peaks are equally distributed over the chromatogram (same resolution) and where, ideally, all irrelevant peaks are confounded with a (hypothetical) $t_{0}$ peak [6]. When these two conditions are fulfilled, $\underline{r}^{*}$ is equal to one. Although this ideal situation is not often met in practice, an important practical consequence of using this type of criteria is that it favors separations where the

\begin{tabular}{ll}
\hline Symbol & Name and mathematical description \\
\hline$S$ & Separation factor \\
& $S_{j i}=\frac{t_{R, j}-t_{R, i}}{t_{R, j}+t_{R, i}}=\frac{k_{j}-k_{i}}{k_{j}+k_{i}+2}$ \\
& Resolution \\
$R_{S}$ & $R_{S, j i}=\frac{S_{j i} \sqrt{\frac{1}{2}\left(N_{i}+N_{j}\right)}}{2}=\frac{S_{j i} \sqrt{\bar{N}}}{2}$ \\
& Effective resolution (lower of following two values) \\
$R_{l}$ & ${ }^{i} R_{n}=\frac{\left(t_{R, j}-t_{R, i}\right)\left(1+A_{S, i}\right)\left(1+A_{S, j}\right) \sqrt{N_{i} N_{j}}}{4 A_{S, i} t_{R, i}\left(1+A_{S, j}\right) \sqrt{N_{j}}+4 t_{R, j}\left(1+A_{S, i}\right) \sqrt{N_{i}} \sqrt{1+0.5 \ln \left(h_{j} / h_{i}\right)}}$ \\
& ${ }^{j} R_{p}=\frac{\left(t_{R, j}-t_{R, i}\right)\left(1+A_{S, i}\right)\left(1+A_{S, j}\right) \sqrt{N_{i} N_{j}}}{4 A_{S, i} t_{R, i}\left(1+A_{S, j}\right) \sqrt{N_{j}} \sqrt{1+0.5 \ln \left(h_{i} / h_{j}\right)}+4 t_{R, j}\left(1+A_{S, i}\right) \sqrt{N_{i}}}$ \\
&
\end{tabular}


Table 2 Overall criteria

\begin{tabular}{ll}
\hline Symbol & Name and mathematical description \\
\hline$c_{\text {min }}$ & Minimum resolution \\
& $C=\min \forall c_{j i}\left(i=1^{\text {a }}\right.$ to $\left.n-1 ; j=i+1\right)$ \\
& Threshold resolution \\
& If $c_{\min } \geq \varepsilon$ then $C=\frac{1}{\left(1+k_{\omega}\right)}$ else $C=0$ \\
& Normalized resolution product $=$ NRP \\
& $C=\prod_{i=1}^{n-1} c_{j i} / \bar{c}$ \\
$r^{*}$ & Calibrated normalized resolution product $=$ CNRP \\
& $C=\prod_{i=0}^{n-1} c_{j i} / \bar{c}$ \\
{$\left[t_{n e}\right]_{f, d}^{-1}$} & Minimum required analysis time (constant flow and particle size) \\
& $C=\frac{c_{\min }^{2}}{\left(1+k_{\omega}\right)}$ \\
{$\left[r_{n t}^{*}\right]_{f, d}$} & Time-corrected resolution product (constant flow and particle size) \\
{$\left[t_{n e}\right]_{p}^{-1}$} & $C=\frac{\sqrt[n]{r^{*}} c_{\min }^{2}}{\left(1+k_{\omega}\right)}$ \\
& Minimum required analysis time (constant pressure drop) \\
{$\left[r_{n t}^{*}\right]_{p}$} & $C=\frac{c_{\min }^{4}}{\left(1+k_{\omega}\right)}$ \\
& Time-corrected resolution product (constant pressure drop) \\
& $C=\frac{\sqrt[n]{r^{*}} c_{\min }^{4}}{\left(1+k_{\omega}\right)}$ \\
&
\end{tabular}

relevant peaks are adjacent and where irrelevant peaks are confounded.

$\underline{r}^{*}=\prod_{k=1}^{d} \underline{c}_{k} / \bar{c}$

with

$\bar{c}=\frac{1}{p} \sum_{i=0}^{n-1} c_{j i}$

where $c$ is the elementary criterion $\left(R_{S}, R_{1} \ldots\right), n$ the number of solutes, $\underline{c}_{k}$ the relevant values of $c$, and $p$ the number of relevant peaks.

The following application illustrates the characteristics of the calibrated normalized resolution product. The optimization of the mobile phase composition (volume fractions of $\mathrm{MeOH}, \mathrm{ACN}$, and THF) for a mixture of nine benzodiazepines is achieved using a classical mixture design (Fig. 2). Figure 5a is the optimum chromatogram selected by $\underline{r}^{*}$ when nordazepam, diazepam, and ethyl loflazepate are relevant. The interesting peaks are adjacent, while irrelevant peaks tend to be confounded (four irrelevant peaks are coeluting: triazolam, oxazepam, flunitrazepam, and clonazepam). Figure 5b shows another example of limited optimization where flunitrazepam, triazolam, and nordazepam are relevant. The peaks of interest are again adjacent in this optimum chromatogram. In comparison with the chromatogram presented in Fig. 5a, where triazolam and flunitrazepam are irrelevant and confounded with two other irrelevant peaks, these two compounds are now separated from the irrelevant peaks. $\underline{r}^{*}$ promotes the separation of solutes into groups. These examples again demonstrate the importance of considering the relevance of the peaks during an optimization strategy.
Fig. 3 Two schematic chromatograms illustrating examples of limited optimization
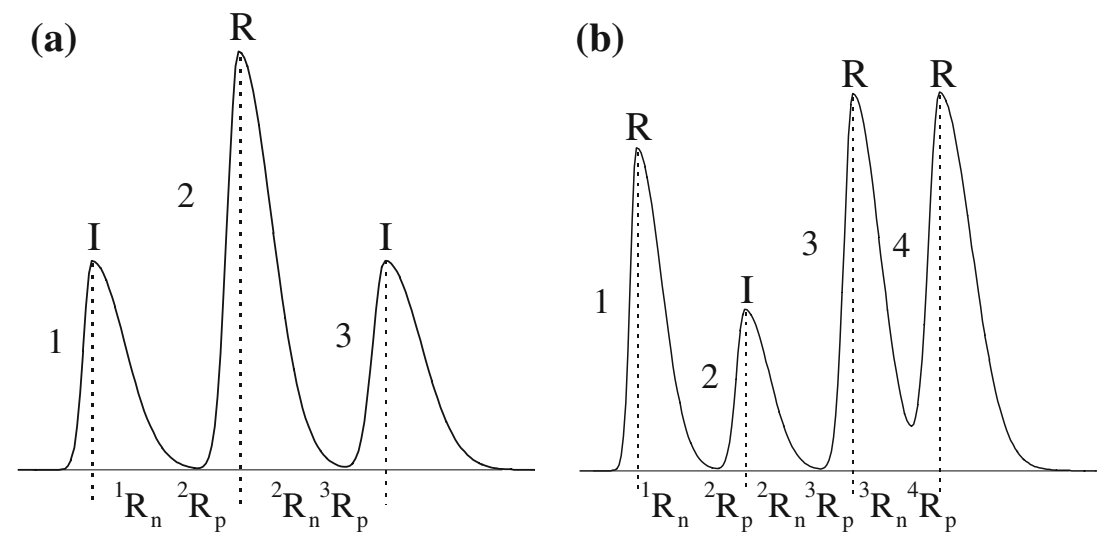
Fig. 4 Optimum chromatograms defined by the minimum effective resolution when a all five solutes are relevant, $\mathbf{b}$ benzoic acid and dinitrobenzoic acid are relevant, and $\mathbf{c}$ only dinitrobenzoic acid is relevant. Peaks: 1 aspirin; 2 3,5-dinitrobenzoic acid; 3 salicylic acid; $4 m$-nitrobenzoic acid; 5 benzoic acid. In chromatograms $\mathbf{b}$ and $\mathbf{c}$, relevant peak numbers are underlined

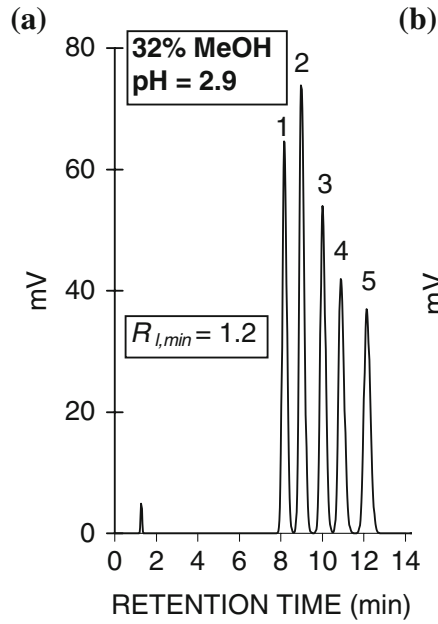

(a) (b)

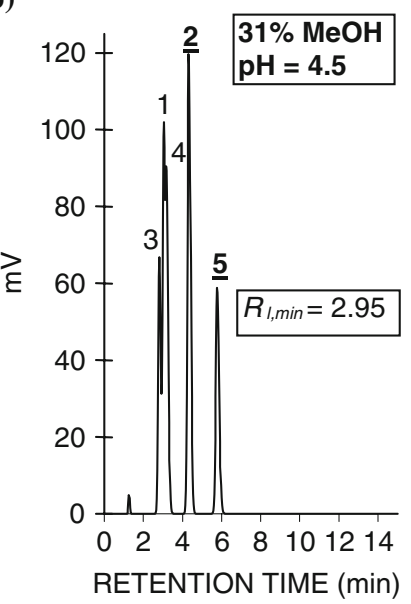

(c)

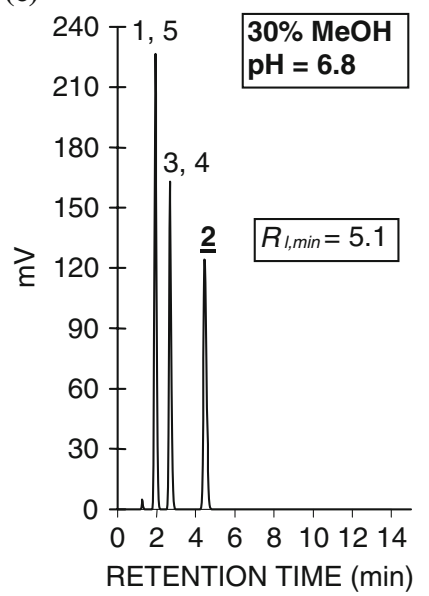

In conclusion, improved optimization criteria which can deal with non-ideal peaks and with limited optimization exist [6], and these should be integrated in all optimization software. Limited optimization is required in many situations and in many application areas because analysts are often interested in the separation of a limited number of solutes in a complex mixture (biological samples, environmental samples, etc.). Good criteria are a vital factor. Different criteria adapted to limited optimization have been described. Among the most useful ones are the minimum (a)

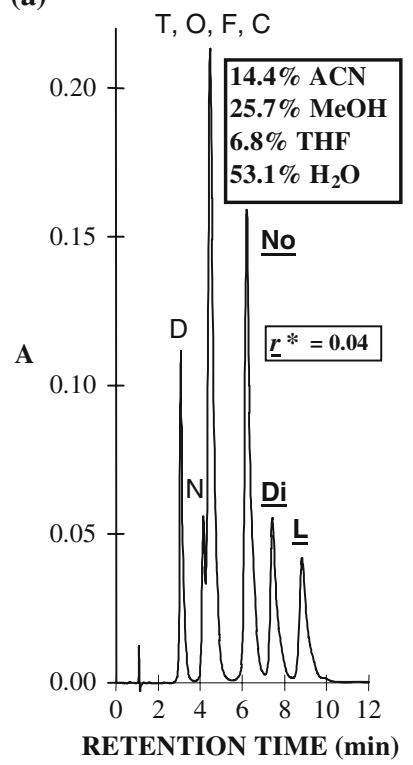

(b)

$\mathbf{A}$

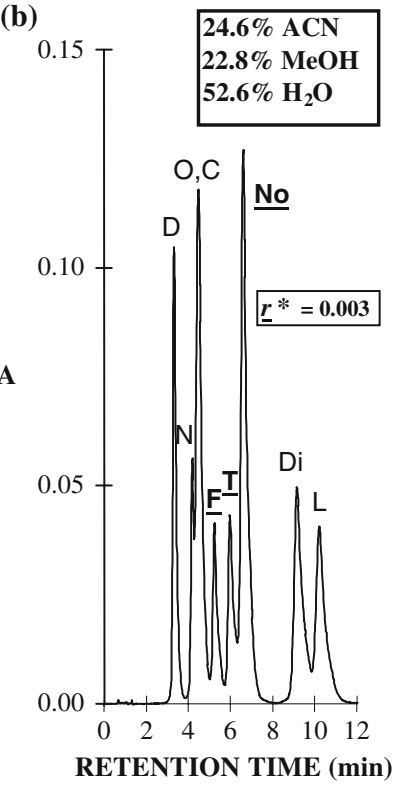

Fig. 5 Optimum chromatograms selected by the calibrated normalized resolution product based on the separation factor when a nordazepam, diazepam, and ethyl loflazepate are the relevant solutes and $\mathbf{b}$ flunitrazepam, triazolam, and nordazepam are relevant. Peaks: $T$ triazolam; $D$ demoxepam; $O$ oxazepam; $F$ flunitrazepam; No nordazepam; $N$ nitrazepam; $C$ clonazepam; $D i$ diazepam; $L$ ethyl loflazepate. Relevant peak numbers are underlined resolution and the calibrated normalized resolution product. The minimum resolution is the obvious choice when the ultimate objective of the separation is to obtain a good resolution between the relevant peaks and all the other peaks. As the minimum resolution does not consider the analysis time, it can lead to lengthy separations. The threshold resolution can be used to avoid this problem. However, the minimum resolution and the threshold resolution do not reflect the distribution of the peaks over the chromatogram. The calibrated normalized resolution product promotes an equal spreading of the relevant peaks throughout the chromatogram. The great advantage of this criterion is that it strongly promotes the co-elution of irrelevant peaks and favors the separation of the solutes into groups. Ideally, the relevant peaks are equally distributed over the chromatogram, and all the irrelevant peaks are confounded with the $t_{0}$ peak. The main disadvantage of normalized resolution products is that high criterion values may be obtained when the actual resolution is low. Therefore, we believe that these criteria, like analysis time, can best be used in a hierarchical, "threshold" format.

In summary, recommended criteria for the limited optimization of separations on a given column are

1. Minimum effective resolution to achieve the best possible separation

2. Threshold resolution to achieve an acceptable separation in the fastest possible time

3. Threshold distribution (i.e., $C=\underline{r}^{*}$ while $R_{l, \min } \geq \varepsilon$, else $C=0$ ) to achieve the best possible distribution of the peaks in combination with an adequate separation

Moreover, the behavior of normalized resolution products adapted to limited optimization opens the way towards the optimization of multidimensional separations and, possibly, group-type separations. The latter is the goal of two-dimensional chromatography. This subject will be discussed latter. 


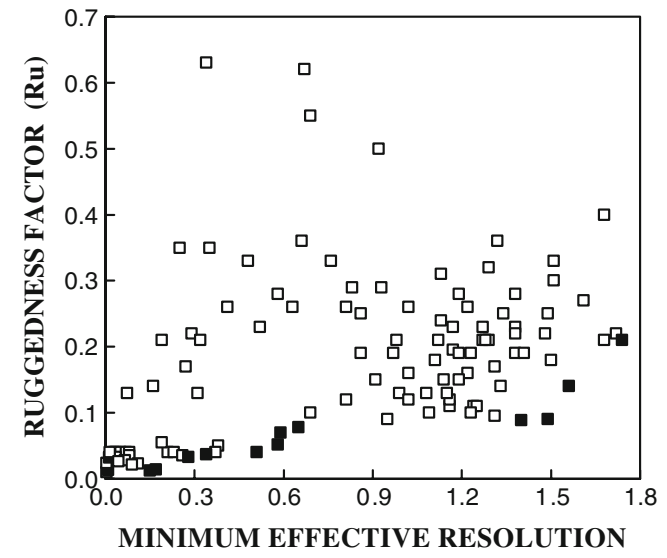

Fig. 6 Example of Pareto-optimality plot obtained during the optimization of the separation of five acidic solutes (see Fig. 1). Optimization criteria are the minimum effective resolution and robustness criterion $R_{u}$. Black squares are the Pareto-optimal points

In the above examples, we demonstrate that it is possible to optimize HPLC analysis without the need of complex algorithm [4, 9, 10]. Indeed, each chromatographic parameter (retention time, peak height, peak area, asymmetry factor, etc.) can easily be calculated without such complex deconvolution algorithms or equations difficult to use in practice.

Another important approach that allows the ultimate robustness of chromatographic methods to be rigorously included as an objective from the outset of systematic method development was described in [7, 8]. In previous work, Vanbel et al. [7] defined such criteria as derivatives of the minimum resolution with respect to the optimized parameters (Eqs. 6 and 7).

$R_{u}=\sum_{i=1}^{n}\left(\Delta_{x_{i}} \frac{d R_{S, \min }}{d x_{i}}\right)$

$\left[R_{u}^{*}\right]^{-1}=\frac{R_{S, \min }}{R_{u}}$

where $R_{S, \min }$ is the minimum resolution, $x_{i}$ the optimized parameter $i$, and $\Delta x_{i}$ the permitted variation of parameter $x_{i}$.

The implementation of multicriteria decision-making techniques is required to find a suitable compromise between robustness and chromatographic resolution (and/ or other objectives such as the analysis time). Figure 6 shows an example of a Pareto-optimality plot for the minimum effective resolution and the robustness criterion, $R_{u}$, obtained during the optimization of $\mathrm{pH}$ and mobile composition for the separation of five acidic solutes [7]. Resolution has to be maximized, and $R_{u}$ has to be minimized. The MCDM plot visualizes directly the pay-off between the two criteria. Information with respect to both criteria is available, so that the chromatographer can decide which of the Pareto-optimal points is preferable (see Fig. 6)

In another study, de Aguiar et al. [8] suggests the use of optimization functions which are multicriteria in nature. Quality of the separation and robustness are combined in the same mathematical equation (Eqs. 8 and 9).

$C R_{1}=n\left(\frac{\left(f_{j}\right)_{S}}{\sum_{i=1}^{n}\left|\frac{\Delta\left(f_{j i}\right)_{S}}{\Delta x}\right|}\right)$

$C R_{2}=\left(\frac{\left(f_{j}\right)_{S}}{\prod_{i=1}^{n}\left(1+\left|\frac{\Delta\left(f_{j i}\right)_{S}}{\Delta x}\right|\right)}\right)$

where $\left(f_{j}\right)_{\mathrm{S}}$ is the scaled response for a point $j$ and $\Delta x$ the variation of parameter $x$.

This approach does not need the use of MCDM techniques to find an appropriate optimum. However, a single number describes the overall quality of the separation, and the chromatographer sacrifices control of the optimization process.

Considering robustness as an objective from the beginning of method development reduces significantly the chance of failure during the validation process. This concept should be systematically integrated in any optimization procedure. Method validation is obviously a highly recommended step.

\section{Two-dimensional chromatography}

Peter et al. [5] proposed a resolution metric for twodimensional chromatography. This resolution measurement is based on the concept of the (one-dimensional) valley-topeak ratio, which has been adapted and modified for twodimensional chromatography. The resolution is calculated using Eq. 10:

$R_{S}=\sqrt{-\frac{1}{2} \ln \left(\frac{1-V}{2}\right)}$

where $R s$ is the resolution in two-dimensional chromatography, and $V$ is the valley-to-peak ratio calculated by Eq. 11:

$V=f / g=\left(g-h_{S}\right) / g$

For additional information, please refer to [5]. The algorithm was developed for GC $\times$ GC. But preliminary studies suggested that its application to other twodimensional separation methods (e.g., LC-LC) should only require minor modification (if any).

New equations to evaluate the separation in twodimensional chromatography should also be derived from 
the equation of the calibrated normalized resolution product (Eq. 4). As explained above, this criterion leads to the separation of the peaks into groups. The calibrated normalized resolution product adapted to limited optimization have the great advantage of strongly promoting the coelution of irrelevant peaks and of favoring the separation of the solutes into groups. The behavior of the calibrated normalized resolution product adapted to limited optimization opens the way towards the optimization of multidimensional separations and, possibly, group-type separations. For example, in two-dimensional separations, relevant peaks do not need to be separated from each other on the first column, but separations in which relevant peaks are not adjacent need to be penalized. In group-type separations, the solutes belonging to a specific group do not need to be separated from each other, but again, situations where solutes of the same group are not adjacent need to be penalized.

Another new concept for two-dimensional chromatography would be to combine resolution and robustness using MCDM techniques. This will be part of future work.

\section{Conclusions}

In this paper, we demonstrate that the selection of adequate optimization criteria is a key step in the success of an optimization process. We show that limited optimization and robustness criteria should be integrated in any optimization procedure. In HPLC, we show that complex deconvolution techniques or equations are often not necessary. In two- dimensional chromatography, more complex optimization strategies have to be implemented. In the latter case, an original approach would be to combine resolution and robustness by using MCDM techniques. This will be part of future work.

Open Access This article is distributed under the terms of the Creative Commons Attribution Noncommercial License which permits any noncommercial use, distribution, and reproduction in any medium, provided the original author(s) and source are credited.

\section{References}

1. Vanbel PF (1999) J Pharm Biomed 21:603-610

2. Vanbel PF, Gilliard JA, Tilquin B (1993) Chromatographia 36:120-124

3. Vanbel PF (1997) Optimization criteria in high-performance liquid chromatography, Ph.D. thesis, Université catholique de Louvain, Bruxelles (B)

4. Vivó-Truyols G, Torres-Lapasió JR, García-Álvarez-Coque MC (2003) J Chromatogr A 991:47-59

5. Peters S, Vivó-Truyols G, Marriott PJ, Schoenmakers PJ (2007) J Chromatogr A 1146:232-241

6. Vanbel PF, Tilquin BL, Schoenmakers PJ (1996) Chemom Intell Lab Sys 35:67-86

7. Vanbel PF, Tilquin BL, Schoenmakers PJ (1995) J Chromatogr A 697:3-16

8. de Aguiar PF, Vander Heyden Y, Massart DL (1997) Anal Chim Acta 348:223-235

9. Vivó-Truyols G, Torres-Lapasió JR, García-Álvarez-Coque MC (2000) J Chromatogr A 876:17-35

10. Martínez-Pontevreda JA, Pensado L, Casais MC, Cela R (2004) Anal Chim Acta 515:127-141

11. Torres-Lapasió JR, García-Álvarez-Coque MC (2006) J Chromatogr A 1120:308-321 